\title{
Viewpoint
}

\section{Foramen-fallacy: Descriptive-delusion}

\author{
Manu Kothari, Atul Goel ${ }^{1}$ \\ Departments of Anatomy and ${ }^{1}$ Neurosurgery King Edward Memorial Hospital \& Seth G.S. Medical College, Parel, Mumbai, India
}

Adi Shankarachrya, in the $9^{\text {th }}$ century AD, and Nicolai Copernicus half-a-millenium later, declared sunrise-nsunset as deceptions. We all - including the media continue to swear by these terms, robbing us all in the bargain of humbling but vivid conceptual change that the sun neither sets nor rises, nor even does the earth, but it is our cussed ingenuity that has been fooling us all along.

Seeing is believing, but believing sans thinking, is deluding. The various foramina of the animal body are a case in point. The discerning Skinner in The origin of Medical Terms traces the term foramen to forare meaning "to bore a hole, to pierce". The all-time great Gray's Anatomy goes on a descriptive rampage: "A hole in bone is a foramen; foramina are canals when lengthy. Large holes may be called apertures or, if covered largely by connective tissue, fenestrae. Clefts in or between bones are fissures".

The whole trouble about the above, Galen-old, description is that it is nowhere near truth. Gray's synonymy between foramen-n-hole allows you to conclude that whatsoever that "passes" through a particular foramen, does so by boring a hole through the bone, or by piercing it. The confusion is worse confounded when Gray describes the "intervertebral foramina" as "the principal routes of entry and exit to and from the vertebral canal." The didactic delusion - that nerves/vessels "run through" a foramen - gets dynamically confirmed by Gray telling that "the facial nerve emerges from the stylomastoid faramen". Bones, it would seem, are already there when nerves/blood vessels arrive, which having done so "enter" into and "emerge" from the bony hole. Ditto delusion for muscles which have their "origin" in one bone and "insertion" in another.

All the modern texts on gross anatomy ail from descriptive compulsions demanding conceptual and terminological delusions. The fait accompli adult cadaver is taken as the basis of all descriptions, thereby giving a short shrift to the actual epigenetic sequence. How about, as a starting point, mulling over this statement? The foramen ovale forms round the mandibular nerve, and the foramen magnum round the neural axis. No foramen worth its name is an exception to this, very ordinary, embryological and epigenetic sequence.

The above Copernican change is a plea that the ease, elegance and the profundity of Nature's working need not be dismissed by our unappreciative temper, paucity of imagination, and poverty of parlance. Slack, in his mini-classic From Egg to Embryo (Cambridge Univ. Press 1983) makes a humbling generalization: "Embryogenic development is terribly complicated. It is a historical sequence of hierchical decisions by which one state of system leads to another". The reality of vertebrate embryogenesis is that the developing body lays down the cables-n-conduits first, followed by their being surrounded/packed/cushioned by connective tissue that, after the full embryogenesis is over by the $8^{\text {th }}$ IUL in humans, gets mineralized here and there to be called bones, that are a put-on on the prelaid nerves and blood vessels, as also muscles. Not one of them traverses a bone, nor seeks "attachment" to it. But the latterly arrival of bone creates the illusion of a foramina/ canal/ fissure/ fenestration/ attachments. The utter disregard that anatomy texts hold for the "hierchical decision" allows them deluding descriptions. Profound poetry gets replaced by pedestrian prose.

An easy metaphor for the laying down of bone around any nerve/vessel is that of a sleeve. A sleeve, from sliv/ slev/sloof/sloove meaning to cover or to put on, looks like the most appropriate replacement for the illogical term foramen etc. The foramen magnum is an occipital sleeve around the neural axis, and so is any bony canal or bony fissure allegedly allowing a structure to pass through it. To render matter bonily more complete, all connective-sleeves that are ossified be called ossleeves or osseous sleeves.

A foramen is a ring sleeve, a fissure a flat sleeve, a short canal a short sleeve, and a long one a long sleeve. 
The endeavor of eusemantics aims at pertinent parlance clearly based on principles. An eusemantic term should be euphonius, and evocative. The moment you see an intervertebral foramen as a sleeve, the mind takes you effortlessly to the primacy and hierarchical priority of a spinal nerve, and the subsequent, second-thought emergence of the bony cover in the post-embryonic fetal phase. So the shift from foramen to sleeve is embryologically and anatomically epigenetic and educative.

The sleeve-magnum (hitherto, foramen magnum) is NOT the largest. The largest is sleeve-maxima, and is located in the ring of the atlas. Next is the occipital sleeve-magnum. The so-called spinal canal represents sleeve-media, the intervertebral cover the sleeve minima and the other minute sleeves should be known as sleeve-miniscula. Each of them is a put-on, an apparel, a bangle, a wrist-watch, a cuff, but none is a foramen.

That the anatomic descriptive fallacies are not just foramen-oriented, can be realized from the fact that the esophagus does NOT pass from the neck, into the thorax, to emerge into the abdomen, nor does the aorta pass through thorax to burst out in the abdomen, but that the so-called neck, thorax, and abdomen get fashioned around hierchically prioritized oesophagus and the aorta.

The all-too-common fallacy of giving certitude to a logically wrong sequence and then confounding it by "bare-faced empiricism and embarrassingly silly terminology" (Nobelist Medawar's lament on immunology) may be called CIFOTHism - the Cart in Front Of The Horseism. CIFOTHism makes you look learned but leaves you cocooned by confusion. The extant descriptions of the anatomy and physiology of the locomotor system are CIFOTHismic, bereft as they are of self-evident epigenesis. The reader is urged to go back to Gray on the facial nerve (vide supra) and rethink, and then reread, the same as follows: "In the neck, the facial nerve turns visible just beyond the stylomastoid sleeve." Correct embryologically, epigenetically, anatomically, and didactically.

Accepted on 19-11-2008

Source of Support: Nil, Conflict of Interest: None declared. 